\title{
Interpolation of the Mean Anomalies for Cloud-Filling in Land Surface Temperature (LST) and Normalized Difference Vegetation Index (NDVI)
}

\author{
Ana F. Militino, M. Dolores Ugarte, Unai Pérez-Goya, and Marc G. Genton
}

\begin{abstract}
When monitoring time series of remote sensing data, it is advisable to fill gaps, i.e., missing or distorted data, caused by atmospheric effects or technical failures. In this work, a new method for filling these gaps called interpolation of the mean anomalies (IMA) is proposed and compared with some competitors. The method consists of: 1) defining a neighbourhood for the target image from previous and subsequent images across previous and subsequent years; 2) computing the mean target image of the neighbourhood; 3 ) estimating the anomalies in the target image by subtracting the mean image from the target image; 4) filtering the anomalies; 5) averaging the anomalies over a predefined window; 6 ) interpolating the averaged anomalies; and 7) adding the interpolated anomalies to the mean image. To assess the performance of the IMA method, both a real example, and a simulation study are conducted with a time series of MODIS TERRA and MODIS AQUA images captured over the region of Navarre (Spain) from 2011 to 2013. We analyze the land surface temperature (LST) day and night, and the normalized difference vegetation index (NDVI). In the simulation study seven sizes of artificial clouds are randomly introduced to each image in the studied time series. The square root of the mean squared prediction error (RMSE) between the original and the filled data is chosen as an indicator of the goodness of fit. The results show that the IMA method outperforms Timesat, Hants, and Gapfill in filling small, moderate, and big cloud gaps in both the day and night LST and NDVI data, reaching RMSE reductions of up to $23 \%$.
\end{abstract}

Index Terms-Geostatistics, MODIS, Smoothing images, Thinplate splines

\section{INTRODUCTION}

Removing clouds from satellite imagery is an important and crucial task for reconstructing the history and evolution of many remote sensing data. While very cloudy images must be dropped from time series, missing or distorted data in images that are only partially clouded can be filled using series of

Ana F. Militino is with the Department of Statistics, Computer Science and Mathematics, and InaMat at the Public University of Navarre, 31006 Pamplona, Spain e-mail:militino@unavarra.es (see http://www.unavarra.es/personal/amilitino/indexfotoingles.htm).

M. Dolores Ugarte is with the Department of Statistics, Computer Science and Mathematics, and InaMat at the Public University of Navarre, 31006 Pamplona, Spain

Unai Pérez-Goya is with the Department of Statistics, Computer Science and Mathematics, and InaMat at the Public University of Navarre, 31006 Pamplona, Spain

Marc G. Genton is with King Abdullah University Science and Technology (KAUST), Computer, Electrical and Mathematical Sciences and Engineering Division (CEMSE), Thuwal, 23955-6900 Saudi Arabia.

Manuscript received June -, 2018; revised — -, 2018. multi-temporal images. Therefore, cloud-filling techniques are frequently used when monitoring features that change over time, such as snow cover [1], [2], vegetation [3], [4], land cover change [5], [6] or forestry [7]. Several procedures have been recently introduced [8], [9], [10], [11], [12], but some of the most popular, e.g., Timesat [13] based on filtering, Hants [14], [15] based on harmonic analysis of time series, and Gapfill [16], [17] based on a specific ordering of images and quantile regression, provide free access to users and are easy to run. However, these gap-filling techniques are neither simple nor straightforward.

In this paper, we present a new method for filling the gaps caused by missing or distorted data, called interpolation of the mean anomalies (IMA). This method uses the same neighbourhood of the target image than Gapfill to capture the temporal dependence between close images, but Gapfill uses quantile regression over the image ranks for predicting every missing data, while IMA uses this neighbourhood to derive the anomalies from the mean image, and interpolate them. The neighbourhood frame is defined as the set of previous and subsequent images in time periods and years, accommodating the temporal dependence between near images, in the same or different years. In IMA, anomalies in the target image are derived from the mean, and averaged over a predefined window after trimming the extreme values. Next, the method interpolates the averaged anomalies in the original resolution over the study region with thin-plate splines (Tps). These interpolated anomalies are added to the mean image to fill in the gaps. The flowchart in Figure 1 summarizes the IMA process for one image.

In this paper, we choose bivariate Tps as the interpolator, because of its simplicity and well-known properties [18]. It is frequently used to calibrate, enhance or improve the quality of remote sensing data [19], [20], [21], though other interpolators are also available [22], [23]. The bivariate thin-plate spline has the advantage that it can be recast as a kriging method with trend of first order, and the generalized covariance function, $\boldsymbol{\Sigma}(d)=|d|^{2} \log (d)$, where $d$ is the Euclidean distance [24], [25]. However, Tps does not need to estimate the variogram parameters as kriging. Nevertheless, it provides similar performance [18] based on the principle that nearby observations tend to be more alike [26].

The idea of using kriging methods to recover gaps in satellite images is not new. In the past, an indicator kriging was used 


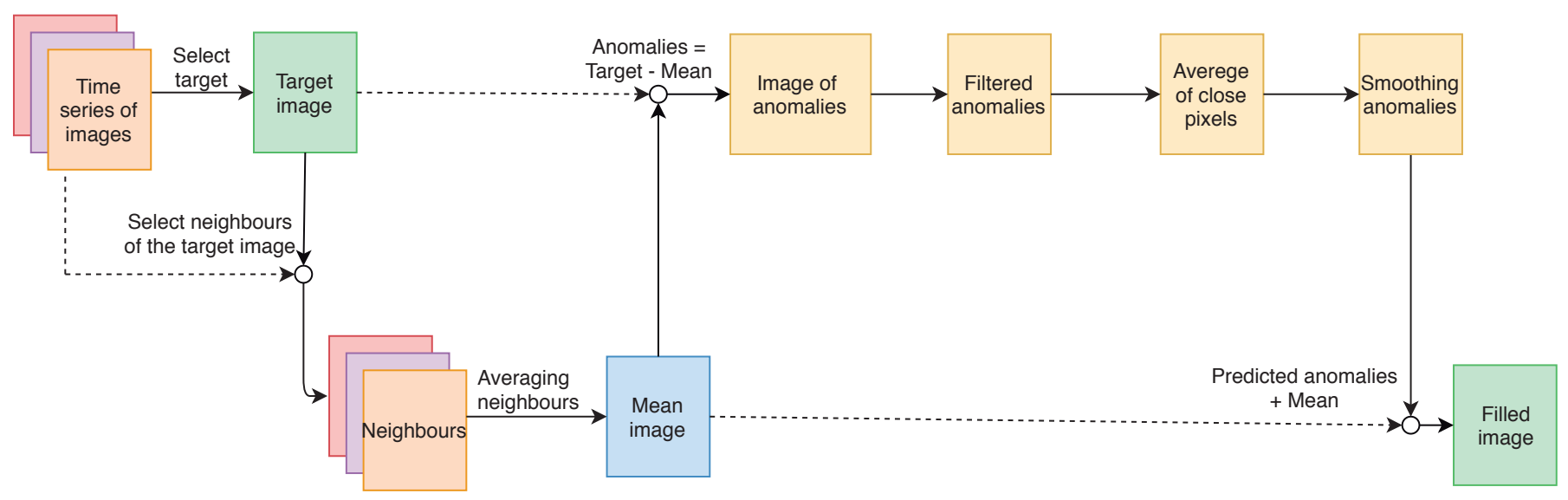

Fig. 1. Flowchart of IMA for processing one image.

to fill Landsat images from Mexico [27]. In that study the author used a kriging method, but the focus of the paper was on calculating the probability that a pixel would be properly classified as obscure or missing remote sensing data, rather than on filling gaps.

Several studies have shown the benefits of using geostatistical methods, over mathematical ones, for filling gaps in satellite imagery. Geostatistical methods explicitly assume that the stochastic spatial dependence inherent in spatial data decreases with distance. For example, [28] made a comparison of the conventional mathematical and the geostatistical methods to replace clouded pixels in NOAA-AVHRR images. The conventional method is maximum value composition (MVC) and the geostatistical methods are kriging and co-kriging. The geostatistical methods performed better than those ignoring stochastic spatial dependence, though co-kriging methods have computing restrictions when applied to large datasets. Some years later, another study compared noise-reduction NDVI model-based methods [29]. In this case, the authors showed that the double logistic and asymmetric Gaussian functionfitting methods of Timesat outperformed the other four alternatives: $4253 \mathrm{H}$ [30], twice filter [30], mean-value iteration filter [31] and ARMD3-ARMA5 filter [32].

The effectiveness of Hants was evaluated [33] by reconstructing the spatial patterns of normalized difference vegetation index (NDVI) time series, and other land surface variables, but only the accuracy of the procedure with regard to MODIS products was shown. Another stochastic approach [34] on spatio-temporal modeling is based on generalized additive models. The application is helpful for situations with high percentages of missing data, but it needs specific programming, and it is very slow when managing large amounts of data.

In response to the 2003 failure of the Scan-Line Corrector (SLC) of the Landsat 7 Enhanced Thematic Mapper Plus (ETM+), some filling gaps contributions were developed. For example, the Neighbourhood Similar Pixel Interpolator (NSPI) [35] was proposed for the aim of filling gaps in satellite imagery. NSPI was later improved using geostatistical techniques [36], [37], and the direct sampling method [38]. Other relevant contributions for filling gaps include the use of differential equations [39], and the estimation of phenological parameters [40]. The success of these models was dependent on tuning some parameters according to the number of annual growing seasons. Geostatistical procedures were also recently compared with direct sampling and weighted regression for cloud-filling in Landsat 7 [41].

Another study [42] compared a variety of approaches to filling the gaps in time series of vegetation parameters. A Fourier-based approach, a double logistic model, an iterative interpolation for data reconstruction, the Whittaker smoother, the Savitzky-Golay filter, and the locally adjusted cubic spline capping were compared, where cubic spline capping is the best for gap filling [43]. The authors used the historical mean over the past 15 years as a benchmark, and they compared random pixels with this reference, instead of the real observed variable. In this paper, the performance of IMA is checked with regard to five alternatives: Hants, Gapfill, and three version of Timesat in both a real example, and a simulation study. Figure 2 shows the flowchart of the simulation study. In the first step we download the time series of the three variables: 72 composite images of normalized difference vegetation index (NDVI), 138 composite images of daytime land surface temperature (LST), and 138 nighttime LST captured from MODIS [44] in the Spanish region of Navarre between 2011 and 2013. Second, we define the seven sizes $(A, B, C, D, E, F, G)$ of the artificial clouds randomly introduced to each image in the time series. Third, we run the five aforementioned alternatives of cloudfilling methods and IMA. Fourth, we calculate the root mean squared prediction error (RMSE) for each gap, and finally, we average the RMSE by year and method in each remote sensing data, and we explain the conclusions using a collection of plots and tables.

The paper is organized as follows. Section 2 describes the MODIS data to be used in the paper. Section 3 provides the explanation of the new IMA method, and a summary of some popular free access alternatives: Hants, Timesat, and Gapfill. It includes several subsections for describing its main features and requirements. Section 4 presents the results obtained in the simulation study. Comparisons are made by plotting the square root of the mean squared prediction error for each variable versus the size of the introduced random clouds. 


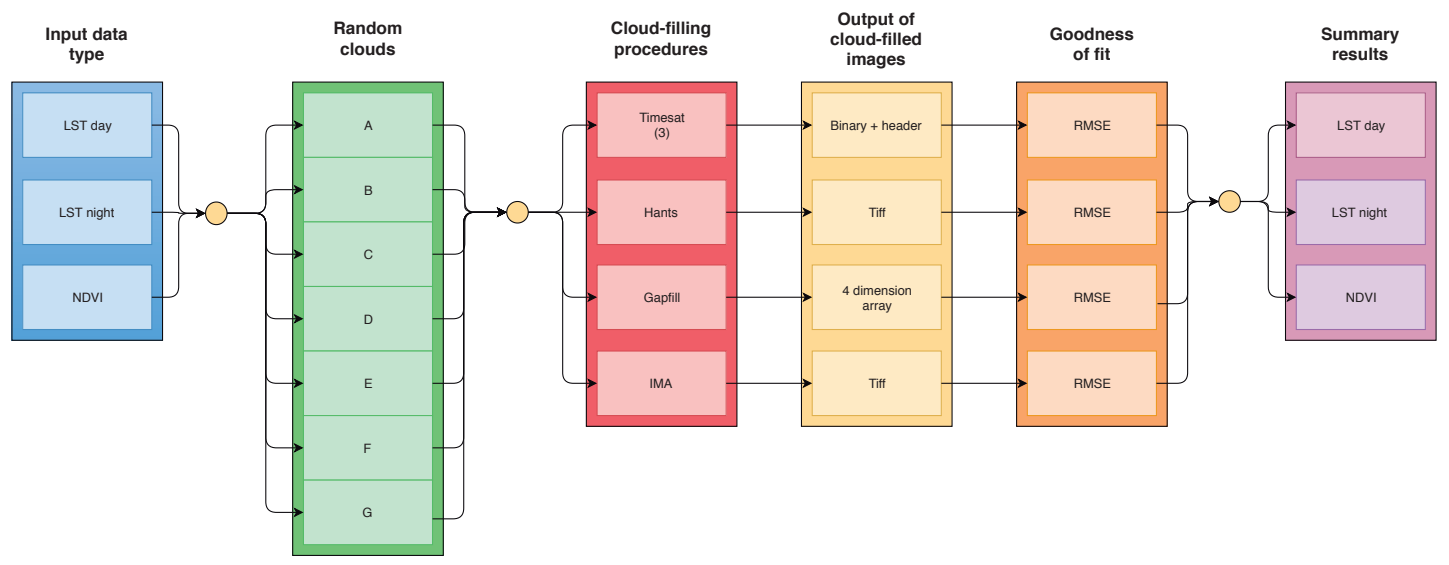

Fig. 2. Flowchart for the simulation study.

Section 5 illustrates the IMA procedure with real data. Section 6 provides a discussion, and finally Section 7 ends with the conclusions.

\section{MODIS DATA}

Frequently, many raw satellite images are almost unusable, because atmospheric disturbances and electronic radiation from the satellites can distort, blur, or degrade the information. The Moderate Resolution Imaging Spectroradiometer (MODIS) [44] provides time series of pre-processed series of images, where these effects have been mitigated. Additionally, image transformations are made to provide very popular remote sensing data, such as NDVI or LST to the users. These time series of images are already enhanced by composing pre-processed images every 8 or 16 days, and they are available for free. We retrieved remote sensing data from MODIS instrument in TERRA and AQUA satellites. NDVI images are of 16-day temporal resolution, then only 23 images are available each year in TERRA, and the same number in AQUA. To adjust the balance with the same number of images every month, we get 23 images from Version 5-MYD13A2 (AQUA), and we retrieve an additional image from Version-5 MOD13A2 (TERRA) in November, yet this is not a prerequisite for running IMA.

The NDVI reflects vegetation vigour and it is closely related to the amount of photosynthetically absorbed active radiation as indicated in [45] and [46]. It is calculated through the radiometric information obtained for the red $(\mathrm{R})$ and near-infrared (NIR) wavelengths of the electromagnetic spectrum. Then, the index is defined as $N D V I=((N I R)-R) /((N I R)+R)$ [47], and takes values between 0 and 1 with high variability [48].

The LST images of MODIS are derived from the two thermal infrared (TIR) band channels, $31(10.78-11.28 \mu \mathrm{m})$ and 32 $(11.77-12.27 \mu \mathrm{m})$ [49]. The atmospheric effects are corrected with the split-window algorithm [50], [51]. The algorithm also uses the MODIS Land Cover product (MOD12C1) for correcting the emissivity effects. Composite LST every eight days are downloaded from Version-5 MOD11A2 and they correspond to the eight days average LSTs of the Version-5 MOD11A1 product. In all variables, we cropped the $H 17-V 4$
MODIS tile containing Navarre, to fit the study region. This region consists of a $156 \times 145$ (22620 pixels) rectangular array, where each pixel corresponds to $1 \mathrm{~km}^{2}$ (see Figure 3).

Table @ shows the study variables, the climatological seasons (winter (DJF), spring (MAM), summer (JJA) and fall (SON)), the coefficient of variation, the minimum, the first, second, and third quartiles, and the maximum of the daytime LST, nighttime LST and NDVI variables in the study region from 2011-2013. LST day and night are given in Kelvin degrees, and NDVI has no units with a restricted range between 0 and 0.98. NDVI has greater variability than LST in the four climatological seasons.

TABLE I

REMOTE SENSING DATA (DATA), CLIMATOLOGICAL SEASON (CS), COEFFICIENT OF VARIATION (CV), MINIMUM, QUARTILES AND MAXIMA OF THE DAY AND NIGHT LST, AND NDVI BY CLIMATOLOGICAL SEASONS IN the NAVARRE TILE (SPAIN), DURING 2011-2013.

\begin{tabular}{llrrrrrr}
\hline Data & CS & CV & Min & 1st Q. & Med & 3rdQ. & Max \\
\hline LST Day & DJF & 0.015 & 252.2 & 279 & 281.3 & 283.5 & 297.5 \\
& MAM & 0.020 & 254.3 & 288.8 & 292.9 & 296.4 & 314.9 \\
& JJA & 0.019 & 265.9 & 298.0 & 302.0 & 307.0 & 320.4 \\
& SON & 0.028 & 251.2 & 285.5 & 290.7 & 297.5 & 314.0 \\
\hline LST Night & DJF & 0.012 & 247.2 & 272.4 & 274.3 & 275.9 & 288.5 \\
& MAM & 0.015 & 249.2 & 277.3 & 280.3 & 283 & 293.3 \\
& JJA & 0.012 & 264.0 & 285.9 & 288.4 & 290.6 & 297.7 \\
& SON & 0.018 & 256.9 & 278.6 & 282.1 & 285.5 & 294.6 \\
\hline NDVI & DJF & 0.33 & 0 & 0.36 & 0.48 & 0.60 & 0.95 \\
& MAM & 0.29 & 0 & 0.48 & 0.60 & 0.70 & 0.95 \\
& JJA & 0.40 & 0 & 0.36 & 0.58 & 0.78 & 0.98 \\
& SON & 0.39 & 0 & 0.34 & 0.52 & 0.70 & 0.98 \\
\hline
\end{tabular}

\section{Cloud-Filling Methods}

\section{A. Interpolation of the Mean Anomalies Method (IMA)}

We assume that the target image is an LST image, named LST_day_2011.073, which corresponds to the 8-day composite image of March 13, 2011, over Navarre, Spain (see Figure 3), although any other time period or variable can also be chosen.

This image is represented by the vector $\mathbf{z}_{\mathbf{s} t_{0}}=\left\{z_{s_{i} t_{0}} \mid i=\right.$ $1, \ldots, m\}$, where $z_{s_{i} t_{0}}$ is the remote sensing data observed at location $s_{i}, s_{i} \in \mathbf{s}=\left(s_{1}, \ldots, s_{m}\right) ; m=22,620$ is the total 


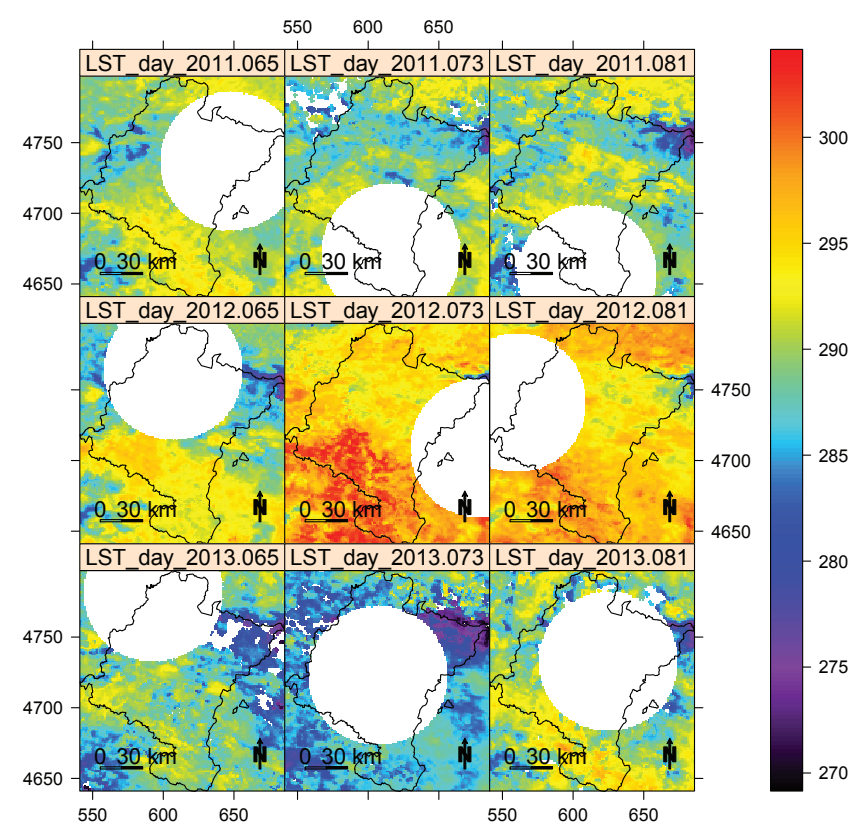

Fig. 3. Example of the neighbourhood of the target image LST_day 2011_073 (color bar units in Kelvin degrees) used in the IMA and Gapfill methods, where random gaps of size $\mathrm{G}$ have been introduced into every image of the neighbourhood. The target image corresponds to the 13 th of March 2011.

number of pixels in the image, and $t_{0}$ is the target time period. Note that $m$ can include pixels of missing data.

The IMA method consists of the next seven steps.

1) Define the neighbourhood of the target image. The neighbourhood $\left\{\mathbf{z}_{\mathbf{s} t_{k}} \mid t_{k}=1, \ldots, T_{0}\right\}$ of the target image $\mathbf{z}_{\mathbf{s} t_{0}}$, consists of the target image, and the preceding and following images in that year, as well as images from those dates during the previous and subsequent years. If the image belongs to the first or last year of the study period, we can choose more subsequent or preceding years, as it is done in this example. Here, the time series are made of eightday composite images of daytime LST data collected from 2011 to 2013. Therefore, the neighbourhood of the target image, including this image has $T_{0}=9$ images, which are identified by the year and Julian day on which they were pre-processed: $I_{1}=2011.065, I_{2}=2011.073, I_{3}=$ $2011.081, I_{4}=2012.065, I_{5}=2012.073, I_{6}=2012.081$, $I_{7}=2013.065, I_{8}=2013.073$, and $I_{9}=2013.081$. See Figure 3 for details. We use the same neighbourhood whether the target image is $I_{2}=2011.073, I_{5}=2012.073$ or $I_{8}=2013.073$ since this study only includes data from 20112013.

The size and dimension of this neighbour can be enlarged according to the availability, the quality of satellites images, and the repetitive cloudiness.

2) Compute the mean target image of the neighbourhood. We assign to each pixel, the mean of the non empty pixels at the same location of the neighbour images. Thus, the mean target image is given by $\overline{\mathbf{z}}_{s t_{0}}=\left\{\bar{z}_{s_{i} t_{0}} \mid i=1, \ldots, m\right\}$, where

$$
\bar{z}_{s_{i} t_{0}}=\frac{\sum_{t_{k}=1}^{T_{0}} z_{s_{i} t_{k}}}{T_{0}}
$$

$z_{s_{i} t_{k}}$ is the ith observed pixel of the $t_{k}$ period in the neighbourhood of the target image.

3) Estimate the anomalies of the target image by subtracting the mean image from the target image. In other words, $\mathbf{w}_{s t_{0}}=$ $\mathbf{z}_{s t_{0}}-\overline{\mathbf{z}}_{s t_{0}}$ is the target image of the anomalies, and $\mathbf{w}_{s t_{j}}=$ $\left\{w_{s_{i} t_{j}} \mid i=1, \ldots, m\right\}$, where

$$
w_{s_{i} t_{j}}=z_{s_{i} t_{j}}-\bar{z}_{s_{i} t_{0}}, \quad \text { for } \quad i=1, \ldots, m .
$$

4) Filter the anomalies. The target anomalies are filtered out by removing the upper and lower $5 \%$ of the extreme values, i.e., percentiles $\left(p_{0.95}\right)$ and $\left(p_{0.05}\right)$ respectively. Removing extreme anomalies prevents from distorted data still present in some images. This step follows the expression

$w_{s_{i} t_{0}}^{\prime}=\left\{\begin{array}{lr}w_{s_{i} t_{0}}, \quad \text { if } & p_{0.05}\left(w_{s_{i} t_{0}}\right)<\left(w_{s_{i} t_{0}}\right)<p_{0.95}\left(w_{s_{i} t_{0}}\right) \\ \text { non value, } & \text { otherwise }\end{array}\right.$

for $i=1, \ldots, m_{1}$. Filtering the target anomalies reduces the maximum total number of pixels in the tile from $m=22,620$ to $m_{1}=20,358$. Alternative threshold values of filtering may be used depending on the quality of the input images, yet these percentiles are recommended.

5) Average the anomalies over a predefined window. The anomalies are averaged over their neighbouring pixels in a window of $5 \times 5$ pixels, when the spatial resolution is 1 $\mathrm{km}^{2}$. We assign the mean of the non-empty anomalies in the same window to all the pixels of the window. Unless all the anomalies in the same window have missing data we will reduce the number of missing pixels. There are three benefits gained by performing this step: we avoid sudden changes among close pixels, we reduce the number of empty 
anomalies, and we reduce the number of the equations to be solved when using Tps. Therefore, a good trade-off between computing time and prediction error is achieved. Removing sudden changes among close pixels is especially important in NDVI images, because these remote sensing data are very sensitive to small changes in vegetation, sensor calibration, and atmospheric correction. Alternative shrinking factors can also be used, depending on the image resolution and computing capacities. Here, we have chosen a factor equal to 5 because the spatial dependence in these variables is about $5 \mathrm{~km}^{2}$, yet it can be changed. Then, the averaged anomalies are defined as

$$
w_{s_{i} t_{0}}^{\prime \prime}=\frac{\sum_{s_{i} \in T_{1}} w_{s_{i} t_{0}}^{\prime}}{25}
$$

where $i=1, \ldots, n, T_{1}$ is a $5 \times 5$ pixel window around $s_{i}$. This step further reduces the $m_{1}$ maximum number of pixels in the target image to $n=m_{1} / 25 \approx 928$ pixels.

6) Interpolate the averaged anomalies. We choose bivariate thin-plate splines to interpolate the target image of the averaged anomalies because of its well known properties [52], yet other alternatives can be used [53]. The thin-plate spline model provides a flexible relationship between the anomalies $\mathbf{w}_{\mathbf{s} t_{0}}^{\prime \prime}=\left(w_{s_{1} t_{0}}^{\prime \prime}, \ldots, w_{s_{n} t_{0}}^{\prime \prime}\right)$, and the the planar coordinates $\left(\mathbf{x}_{\mathbf{s}}, \mathbf{y}_{\mathbf{s}}\right)$. Predictions are given by $\hat{\mathbf{w}}_{s t_{0}}^{\prime \prime}=\sum_{j=1}^{3} a_{j} p_{j}\left(s_{i}\right)+$ $\sum_{i=1}^{n} b_{i} \phi(d)$, where $\phi(d)=d^{2} \log (d)$ is a basis function, $d$ is the Euclidean distance between the prediction location $s_{0}$, and each data location $s_{i}$, and $p_{1}\left(s_{i}\right)=1, p_{2}\left(s_{i}\right)=x, p_{3}\left(s_{i}\right)=y$. The weights $\left\{a_{j} \mid j=1,2,3\right\}$ and $\left\{b_{i} \mid i=1, \ldots, n\right\}$ are estimated by solving a linear system of order $n$ [54], [55], [56]. The predictions are obtained over the $m$ pixels of the target image. This process is nowadays programmed in mathematical, remote sensing, and statistical software in a very efficient way. Here, we use the $R$ package fields [57], where uncertainty measures can also be derived.

7) Add the interpolated anomalies to the mean image. Thus, the final predicted image is

$$
\hat{\mathbf{z}}_{\mathbf{s} t_{0}}=\overline{\mathbf{z}}_{\mathbf{s} t_{0}}+\hat{\mathbf{w}}_{\mathbf{s} t_{0}}^{\prime \prime}
$$

Finally, we programmed IMA in R; and the code for running IMA is available from the authors.

TABLE II

NUMBER OF FILLED IMAGES FOR THE THREE REMOTE SENSING DATA (LST DAY, LST NIGHT AND NDVI), TIME PERIODS, YEARS, CLOUD SIZES, AND METHODS (HANTS, 3 TIMESAT, GAPFILL, AND IMA) USED IN THE SIMULATION STUDY.

\begin{tabular}{lrrr}
\hline & LST Day & LST Night & NDVI \\
\hline Time periods by year & 46 & 46 & 24 \\
Years & 3 & 3 & 3 \\
Cloud Sizes & 7 & 7 & 7 \\
Number of methods & 6 & 6 & 6 \\
\hline Total & 5,796 & 5,796 & 3,024 \\
\hline & \multicolumn{2}{c}{ Total of 14,616 filled images } \\
\cline { 2 - 3 }
\end{tabular}

\section{B. Hants}

The Harmonic Analysis of Time Series (Hants) was a procedure originally developed for processing time series of noisy remote sensing data [14], and a few years later the Hants algorithm was published [15]. The performance of this algorithm has been studied with applications to leaf area index (LAI), land surface temperature (LST), and the polarization difference brightness temperature (PDBT) [33]. The application was released as plug-in for the geographical information system (GIS) platform called the Geographic Resources Analysis Support System (GRASS)[58]. The Hants algorithm uses an iterative procedure to fit a curve based on pixel-wise time series separately, but ignores stochastic spatial dependence. The process follows the steps:

a) checking the time series and flag samples outside the valid range of data,

b) fitting the remaining valid samples of the series by several prescribed harmonic components,

c) if the maximum signed bias between the fitted series and the valid samples is larger than a user defined threshold, and the number of the remaining samples exceeds the minimum number of samples necessary for the reconstruction process, then it rejects the samples with bias larger than half of the maximum bias and return to step b). Otherwise, stop the processing.

\section{Timesat}

Timesat [13] is a software released in 2002 and coded in Matlab and Fortran, yet those are not necessary for running it. It implements three processing methods based on least-squares fits for satellite time series processing: Savitzky-Golay (SG) filtering, double logistic (DL) and asymmetric Gaussian (AG). The software was designed to analyze satellite time series data from satellites by extracting seasonal parameters from smoothed versions of the data, but it works with pixel-by-pixel time series. Timesat needs a specific image binary format that can be obtained using the raster package of the $R$ software [59]. First, the user must specify the number of rows and columns to be processed $(156 \times 145)$, the type of data (16 bit integer), the range of the variables ([0-10000] for NDVI and [0-999.9] for LST day/night), the lag period length (8 or 15), and the time series length (three years). These configuration input parameters must be provided using a graphical interface. When exporting the smoothed image, a matrix is produced, that must be completed in order to be exported to $\mathrm{R}$; the raster package will help again to convert an $h d r$ format into a Tiff format.

\section{Gapfill}

Gapfill is the specific function of the gapfill $\mathrm{R}$ package [16] that fills missing values of satellite data with the Gapfill method. The method ranks the images preceding and following the target image, and predicts the gap using quantile regression. This regression is an extension of the classical estimation of the conditional mean model to conditional quantile functions, which can be explained as follows.

Let $\mathbf{p}=\left\{p_{i j}\right\}$, for $i=1, \ldots, I$ and $j=1, \ldots, J$ be the $j$ th pixel in the $i$ th image and let $\mathbf{r}=\left\{r_{1}, \ldots, r_{I}\right\}$ be the ranks or ordinal sequence of these pixels. Then, instead of estimating the conditional expectation of the response variable 
$\mathbf{p}$ given the explanatory variable $\mathbf{r}$, i.e., $E[\mathbf{p} \mid \mathbf{r}]$, as it is done in classical linear regression, the quantile regression estimates the conditional $\theta$ quantile given $\mathbf{r}$, i.e., $Q_{\theta}(\mathbf{p} \mid \mathbf{r})$. Therefore, the $\theta$ quantile regression model is defined as

$$
Q_{\theta}(\mathbf{p} \mid \mathbf{r})=\beta_{0}(\theta)+\beta_{1}(\theta) \mathbf{r},
$$

where $\beta_{0}(\theta)$ and $\beta_{1}(\theta)$ are the regression coefficients with a similar interpretation of the classical regression model [60]. This algorithm was explained and compared with Timesat for NDVI in a very recent paper [16]. To run Gapfill in our simulation study, we tune the programming to tackle the challenge of filling big gaps. First, in the Predict function we enlarge to 20 the minimum number of non-empty pixels in the target image, originally written as $n$ TargetImage $=5$. Second, in the rank function we add the argument ties.method $=$ first for avoiding ties in assigned ranks. Third, we include the code if $(\operatorname{sum}($ ! is.na $(r))<2)$; return $(N A)$ to guarantee at least 2 different ranks for running the quantile regression. When many missing pixels are in the same locations of different images in the same neighbourhood, it is not possible to assign ranks. Gapfill is easily accesible because of its free distribution, and it provides measures of uncertainty through prediction intervals.

TABLE III

Cloud Size, RADIUS, TOTAL SURFACE AND MEAN SURFACE PERCENTAGE OF THE DISTORTED IMAGES WITH THE ARTIFICIAL CLOUDS USED IN THE SIMULATION STUDY FOR LST DAY.

\begin{tabular}{crrr}
\hline Cloud size & Radius $(\mathrm{km})$ & Surface $\left(\mathrm{km}^{2}\right)$ & Mean Surface $(\%)$ \\
\hline A & 15 & 706.8 & 5.5 \\
B & 17.5 & 962.1 & 6.6 \\
C & 20 & 1256.6 & 7.6 \\
D & 30 & 2827.4 & 13.0 \\
E & 50 & 7852.9 & 28.4 \\
F & 60 & 11309.7 & 37.1 \\
G & 70 & 15393.8 & 44.4 \\
\hline
\end{tabular}

\section{Simulation Results}

The performance of the proposed IMA method for filling clouds in satellite imagery is evaluated in both a real example and a simulation study using 348 images captured over Navarre, Spain, between 2011 and 2013. In the simulation study, IMA is compared with Hants, the three versions of Timesat, and Gapfill. Time series of daytime LST (LST Day), nighttime LST (LST Night), and NDVI are analyzed. The LST time series each contains 138 composite images of 8-day time periods; the NDVI time series contains 72 composite images of 16-day time periods. In the images of these time series, we introduced artificial clouds of seven different sizes $(A, B, C, D, E, F, G)$ to generate missing data inside a randomly located circle. Figure 3 shows an example of randomly introduced size-G clouds. For each of the images of the time series and variables, we run the six cloud-filling methods mentioned above: Hants, three versions of Timesat,

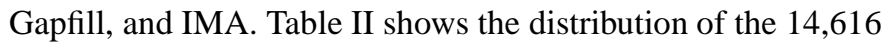
images used in the simulation study according to the derived variables, the cloud sizes, and the methods. Table III shows the cloud size, the radius, the total surface (calculated as $\pi \times r^{2}$ ), and the mean surface percentage of the artificial cloud coverage. The mean surface percentage varies from $6 \%$ to $44 \%$ depending on the size of the cloud, and how much of the cloud is located inside the tile.

We evaluate the performance of the methods for each size of artificial cloud, each derived variable, and each model. Pixelby-pixel square differences between the observed and filled data are averaged for calculating the square root of the mean squared prediction error (RMSE). The expression is given by

$$
\begin{aligned}
R M S E(k, l, p)= & \sqrt{\frac{\sum_{s_{i}, t_{j}}\left(z_{s_{i} t_{j} k l p}-\hat{z}_{s_{i} t_{j} k l p}\right)^{2}}{I T}}, \\
& s_{i}=1, \ldots, I \\
& t_{j}=1, \ldots, T \\
& k=A, B, C, D, E, F, G \\
& l=\text { LST day, LST night, NDVI, and } \\
& p=\{\text { Gapfill, Hants, Timesat AG, } \\
& \text { Timesat DL, Timesat SG and IMA }\},
\end{aligned}
$$

where $z_{s_{i} t_{j}}$ and $\hat{z}_{s_{i} t_{j}}$ are respectively the original and predicted values of the remote sensing data, $I$ is the number of pixels inside the cloud gap, $T$ is the number of images, $k$ is the type of cloud, $l$ is the derived variable, and $p$ is the smoothing procedure.

The $R M S E(k, l, p)$ for the six cloud-filling methods, the seven sizes of artificial clouds, and the three derived variables is shown in Figures 4 and 5 respectively. The left plot in Figure 4 exhibits the LST day RMSE. Different lines correspond to different cloud-filling methods. Pink and black colours are for Gapfill and IMA respectively, while the others colours correspond to Timesat and Hants. In this Figure, Hants and the three Timesat versions show similar RMSE values for the small and moderate cloud sizes, though Hants gives the highest RMSE values for the big clouds. Both Hants and Timesat consistently produce higher RMSE values than Gapfill or IMA. IMA clearly outperforms Gapfill regardless of cloud sizes. Similar conclusions are drawn for LST Night (on the right of Figure 4), where Hants exhibits the highest RMSE values, though not just for big clouds, and IMA always shows the lowest RMSE values for all cloud sizes. Figure [5 shows the RMSE for NDVI. We observe a similar pattern, but we also note a positive correlation between RMSE and cloud sizes. Hants provides the highest RMSE values for almost all the cloud sizes, and IMA the lowest for all the cloud sizes. RMSE estimates are lower for NDVI than for LST because NDVI is constrained to take values between 0 and 1 .

Clearly, Gapfill and IMA outperform the others methods, and therefore, both Tables IV and $\mathrm{V}$ focus on the RMSE estimates and the percent of reduction. Shrinking percentages are always in favor of IMA, however we see in Table [V] that these percentages decrease as cloud size increases, because both methods become less efficient as long as the clouds get bigger. In Table $\mathrm{V}$, we see no such inverse correlation between cloud size and reduction percentage, mainly because NDVI is fairly variable and its values are limited between 0 and 1. Extensive 

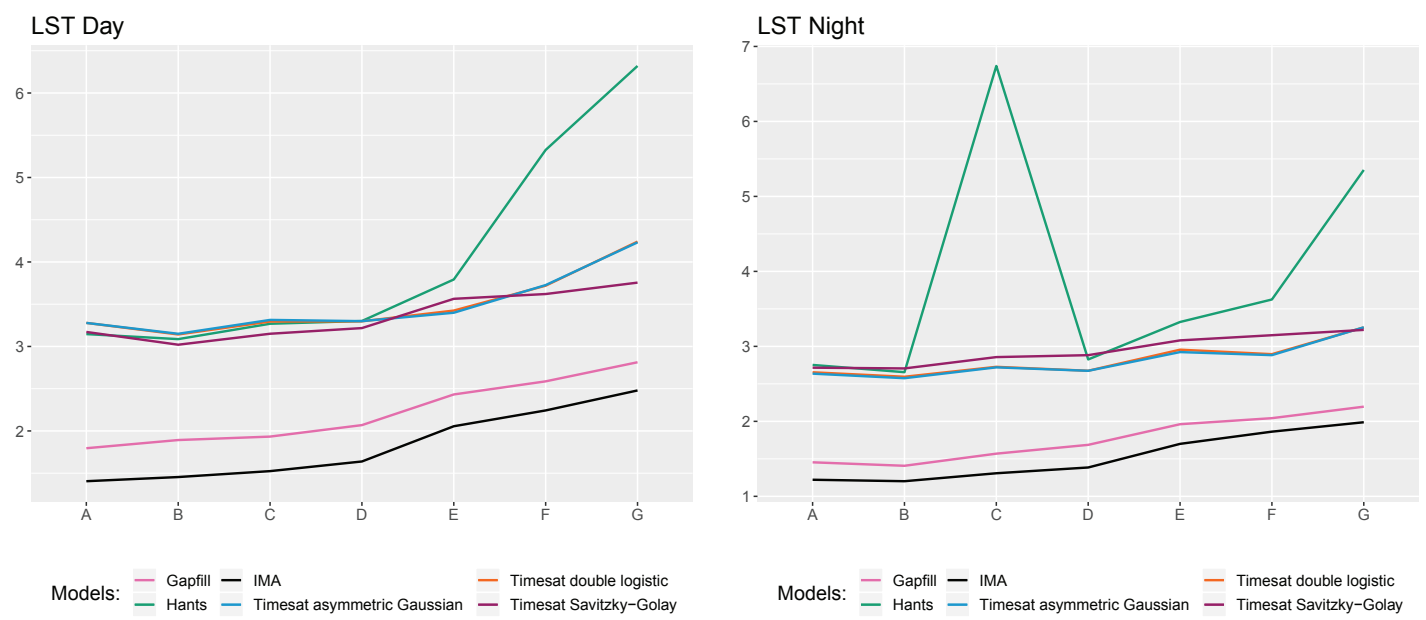

Fig. 4. Root Mean Squared Prediction Error (RMSE) versus artificial cloud size for the six models compared in the simulation study with LST Day (on the left) and LST Night (on the right) images of Navarre, Spain, 2011-2013.

simulation studies not shown here for preserving space reveal that inside a cloud, the pixels closer to the center of the cloud have higher RMSE values than those near the boundary, but the fidelity of the prediction also depends on the similarity of the missing pixels to those from which they borrow information.

TABLE IV

Root Mean SQUared Prediction ERror of GAPFILl (GF) AND IMA, AND REDUCTION PERCENTAGE OBTAINED FROM THE SIMULATION STUDIES OF LST DAY AND LST NIGHT.

\begin{tabular}{l|rrr|rrr}
\hline & \multicolumn{3}{|c|}{ RMSE LST Day } & \multicolumn{3}{c}{ RMSE LST Night } \\
& GF & IMA & Reduction (\%) & GF & IMA & Reduction (\%) \\
\hline A & 1.80 & 1.41 & 21.7 & 1.46 & 1.22 & 16.0 \\
B & 1.89 & 1.45 & 23.2 & 1.41 & 1.21 & 14.6 \\
C & 1.93 & 1.52 & 21.1 & 1.57 & 1.31 & 16.7 \\
D & 2.07 & 1.64 & 20.8 & 1.69 & 1.38 & 17.9 \\
E & 2.43 & 2.06 & 15.5 & 1.96 & 1.70 & 13.4 \\
F & 2.59 & 2.24 & 13.3 & 2.04 & 1.86 & 8.8 \\
G & 2.81 & 2.48 & 11.9 & 2.20 & 1.99 & 9.5 \\
\hline
\end{tabular}

TABLE V

Root Mean Squared Prediction Error of GAPFILl (GF) AND IMA, AND REDUCTION PERCENTAGE OBTAINED IN THE SIMULATION STUDY OF NDVI.

\begin{tabular}{l|rrr}
\hline & \multicolumn{3}{|c}{ RMSE NDVI } \\
& GF & IMA & Reduction $(\%)$ \\
\hline A & 0.051 & 0.046 & 8.5 \\
B & 0.051 & 0.048 & 7.0 \\
C & 0.050 & 0.048 & 4.4 \\
D & 0.063 & 0.055 & 12.4 \\
E & 0.067 & 0.060 & 11.3 \\
F & 0.075 & 0.066 & 11.7 \\
G & 0.082 & 0.073 & 11.0 \\
\hline
\end{tabular}

Overall, IMA outperforms the three versions of Timesat, Hants, and Gapfill regardless of the cloud size for all three variables, LST Day, LST Night, and NDVI. Gapfill is the closest competitor. Figure 7 illustrates the filling processes of the six methods when filling the target image LST_2011_073. Coordinates are given in UTM scaled to $\mathrm{km}$. In this example, IMA provides the best filling.

\section{RESUlTS OF IMA AND GAPFILL PROCEDURES WITH REAL DATA}

Additionally, we also illustrate the IMA procedure in real data by filling clouds in the LST day and NDVI daily target images of the 16th (2012198), 17th (2012199), and 18th (2012200) of July 2012 in Navarre, Spain. Those days were in principle free of clouds, but we mimic cloudy days adding a real cloud mask to the target images.

Daily images are more variable than composite images, because they are only slightly pre-processed. Therefore, for a robust estimation of the anomalies we need to increase the neighbourhood size of the target images. Now, each target image has a neighbourhood of $3 \times 7$ images corresponding to the 3 previous and 3 subsequent images of the same year, and the corresponding images from those dates during the previous and subsequent years. The 3 target images are consecutive, then the neighbourhood is made up of 27 images, and in the end, all the images are filled. The LST day images are daily images retrieved from MOD11A1 (TERRA) Version 5 with a spatial resolution of $1 \mathrm{~km}^{2}$. The NDVI images are defined using the red and near infrared wavelengths from MOD09GA (TERRA) Version 5 in the same days, because MODIS does not provide NDVI daily images. These images are reprojected for homogeneity reasons to the same resolution of LST day images, because the original resolution is $.5 \mathrm{~km}^{2}$, yet this step is not required in the IMA procedure.

The first row of Figure 6 shows the observed LST day target images of the 16th (2012198), 17th (2012199) and 18th (2012200) of July 2012. The second row shows the same images masked with real clouds from the 3th, 14th and 21th of July 2011. The third row shows the high fidelity of the IMA predicted images to the original target ones. Table VI summarizes the root mean squared prediction error obtained with IMA and Gapfill in the clouds of the LST day and NDVI target images. Separately and jointly, IMA reduces the root mean squared prediction error estimated by Gapfill in LST day and it is equally competitive than Gapfill in NDVI images, matching the results of the simulation study. The computing 


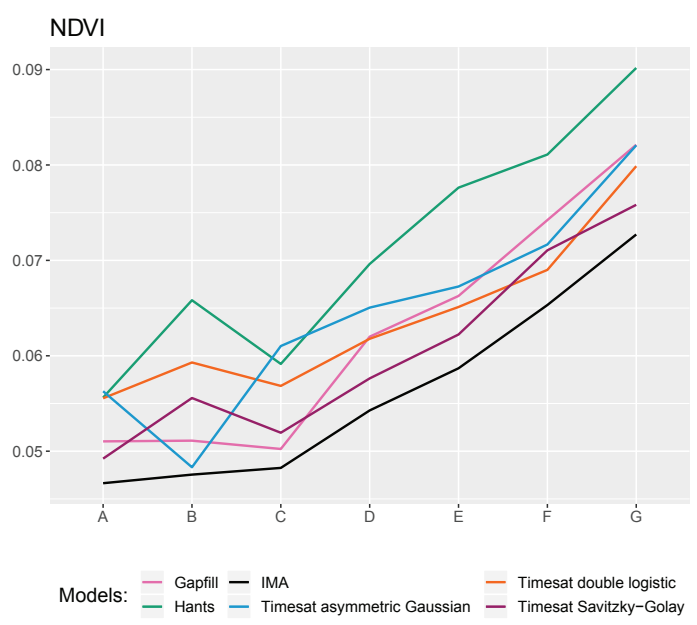

Fig. 5. Root Mean Squared Prediction Error (RMSE) versus artificial cloud size for the six models compared in the simulation study with NDVI images of Navarre, Spain, 2011-2013.

time for processing the raster of the three LST day target images in a PC with an Intel Core i7-4790, and 16GB of RAM takes about 4 seconds with IMA, while Gapfill takes $1 \mathrm{~h} 30$ for filling only the gaps of those images.

TABLE VI

Root Mean SQUared Prediction ERror in the Cloud Set of the LST DAY AND NDVI IMAGES OF NAVARRE OF THE 16TH (2011198), 17TH (2011199) AND 18TH (2011199) OF JULY 2012 OBTAINED WITH GAPFILL (GF) AND IMA.

\begin{tabular}{r|rrrrr}
\hline & Filling cloud & \multicolumn{2}{|c}{ RMSE LST day } & \multicolumn{2}{c}{ RMSE NDVI } \\
Julian Day & Surface $\mathrm{km}^{2}$ & GF & IMA & GF & IMA \\
\hline 2011198 & 7332.53 & 1.58 & 1.28 & 0.07 & 0.07 \\
2011199 & 12081.15 & 4.78 & 1.99 & 0.08 & 0.08 \\
2011200 & 16877.81 & 4.87 & 2.03 & 0.10 & 0.11 \\
\hline sample mean & 12097.16 & 3.74 & 1.77 & 0.08 & 0.09 \\
\hline
\end{tabular}

\section{Discussion}

For checking the performance of IMA with regard to the aforementioned gap-filling methods, an extensive simulation study involving the filling process of 14,616 LST day and NDVI images in different scenarios was conducted. Every gap-filling method is based on different models and assumptions, becoming difficult a theoretical comparison. Therefore, the evaluation of the RMSE is proposed. The RMSE is an indicator of the gap-filling fidelity because it has the same measurement units than the original variable, and it is very intuitive. Nevertheless, it can be calculated only when we know the ground-truth data, because then we can subtract the ground-truth data from the prediction. Simulation procedures make it possible, because we know real data. Furthermore, a data set of 3 target LST day and NDVI images using a neighbourhood of 27 images was created for illustrating the performance of IMA with regard to Gapfill algorithm. Overall, both the simulation study and the real data proved the outperformance of the IMA method.

As for computing time, Table VII shows the running times required for processing 5,796 images. They correspond to 138 different LST Day images processed with all methods, and different sizes of artificial clouds on a Windows PC with an Intel Core i7-4790, and 16GB of RAM. Hants and Timesat are really fast, but Gapfill is slowed-down by its ranking process. The IMA method maintains a constant running time for both small and large gaps in the data, and is faster than Gapfill when processing moderate or large gaps. Though it is not as fast as Timesat or Hants, IMA processes the target images in less than 14 seconds on average, regardless of the gap size.

The filling process of both Timesat and Hants require a moderate number of contiguous images from the same or, if the studied time period is at the very beginning or end of a year, the neighbouring year, but Gapfill and IMA need just a few images from previous and subsequent years to process the target image. However, Timesat and Hants smooth all the images in a row, because all images in the same time series become target images, while Gapfill needs only a neighbourhood to fill one target image, and IMA uses the same neighbourhood for filling several images. Timesat and Hants programs smooth the time series of target images regardless of the presence of gaps or not. However, gaps that must be filled need to be identified prior to processing in Gapfill and IMA. Usually, they are identified as missing data. Gapfill fills the empty pixels of the target image one by one, and IMA fills simultaneously all the pixels of the target image. Gapfill and IMA take profit of the temporal dependence using the next and previous images across the years in a neighbourhood where great similarities are expected. Gapfill incorporates the spatial dependence among remote sensing data when ranking nearby pixels, and IMA when averaging, and interpolating the anomalies. The shortcomings of Timesat and Hants are the tuning constants and the input parameters that must be configured. Their advantages come from their short running time, and the extraction of seasonality parameters made by Timesat.

The IMA procedure also provides the standard errors of the anomalies when adding the option fun=predictSE from fields package [57] in the interpolate command. The approximation is borrowed from the kriging version of Tps function, and it 


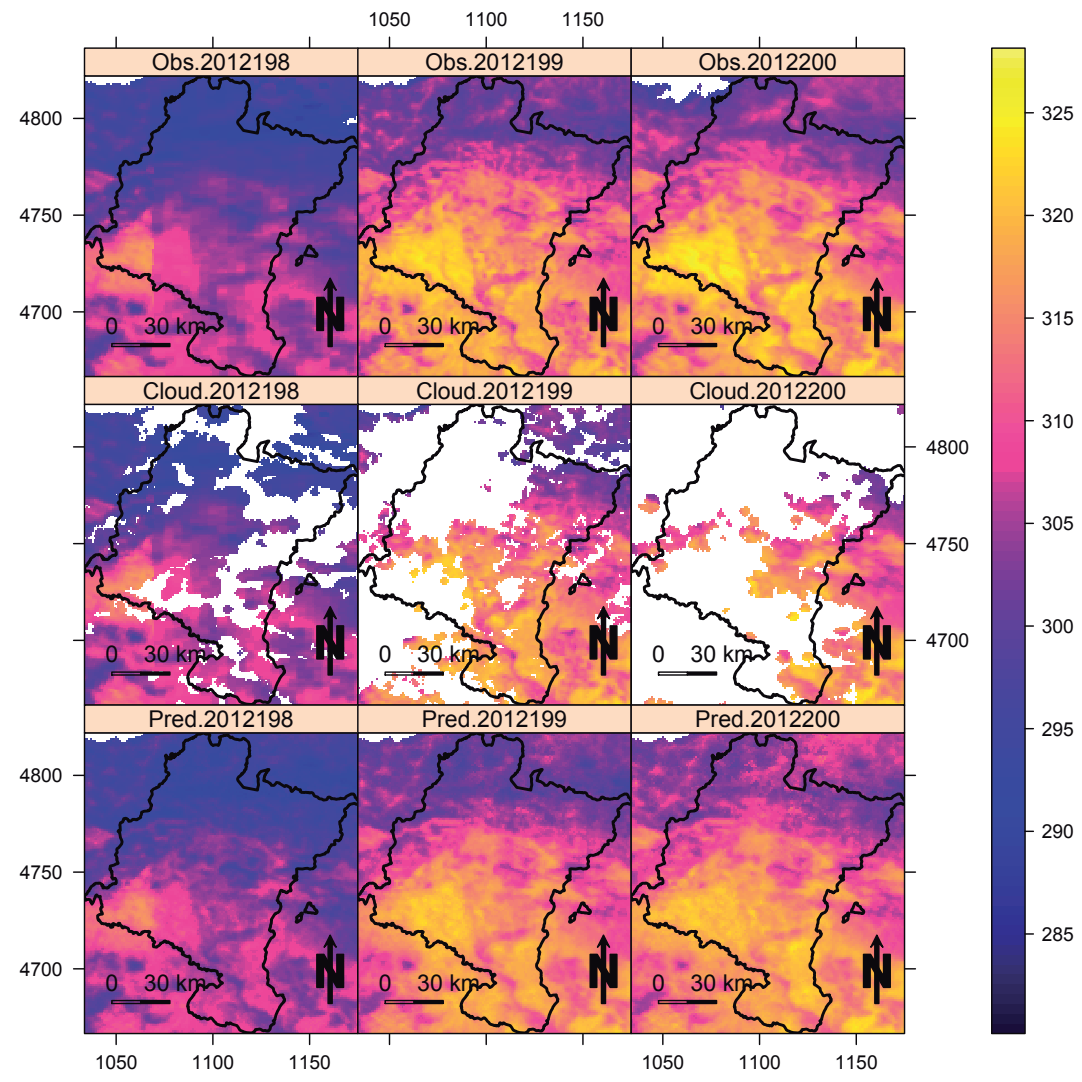

Fig. 6. The first row shows the observed LST day target images (in Kelvin degrees) of the 16th (2012198), 17th (2012199), and 18th (2012200) of July 2012 in Navarre. The second row shows the same images masked with real clouds borrowed from the 3th, 14th and 21 th of July 2011 respectively, and the third row shows the IMA predicted images.

assumes that the covariance parameters are already known. Hence, computing time for running IMA could increase substantially, because standard errors are calculated by default for every pixel of the target image. Processing the 27 images of the real example takes less than 40 seconds, but calculating standard errors of only one image can last 10 minutes in a one thread computing and 5 minutes in a multi-treading computing. The reason could be that the predictSE option is a linear approximation function that is not optimized for rasters. An alternative for reducing it is calculating the standard errors in a lower resolution raster, because estimates are fairly similar across the tile. For example, with an aggregation of factor 8 , IMA takes on average 2 minutes to process the standard errors of one image.

Gapfill authors [16] distinguish 4 steps in the uncertainty estimation, but in the end only a combination of the 5-95 percentile prediction intervals of steps 2 and 3 is provided, because these steps dominate the uncertainty contributions, and adding all steps hardly increase the estimation of the prediction intervals. Computer time of the procedure is slightly increased. Gapfill takes $1 \mathrm{~h} 45 \mathrm{~m}$ for gap-filling 27 images, including the prediction intervals, and can be reduced to $1 \mathrm{~h} 30 \mathrm{~m}$ when it does not estimate them. This time is obtained for a one-thread computing in a PC with an Intel Core i7-4790, and $16 \mathrm{~GB}$ of RAM.
Simultaneous comparison of IMA and Gapfill uncertainty measures is then a difficult task because both procedures provide different uncertainty measures (standard errors and prediction intervals), and the methodologies are completely different.

Cloud-filling methods have some limitations. For example, clouds that are found in the same locations across neighbouring images in a systematic and periodic way could hinder efficient filling of gaps. Larger clouds cause estimation methods to lose robustness and become unstable. In those cases, wider neighbourhoods are required in IMA, and likely programming improvements based on distributed programming and parallelizing are also necessary when input images are of high resolution. The independent steps required for running IMA can be easily fitted in the map-reduce theory [61], and used in Hadoop cluster [62]. We are currently dealing with these issues.

\section{CONCLUSIONS}

The IMA method assumes that remote sensing data can be expressed as the sum of a trend plus a random error. The trend is assumed to be constant in the neighbourhood of the target image, and it is estimated with the mean of this neighbourhood, and the residuals or anomalies are the estimates of the random error. Using repeated measurements from the same and 


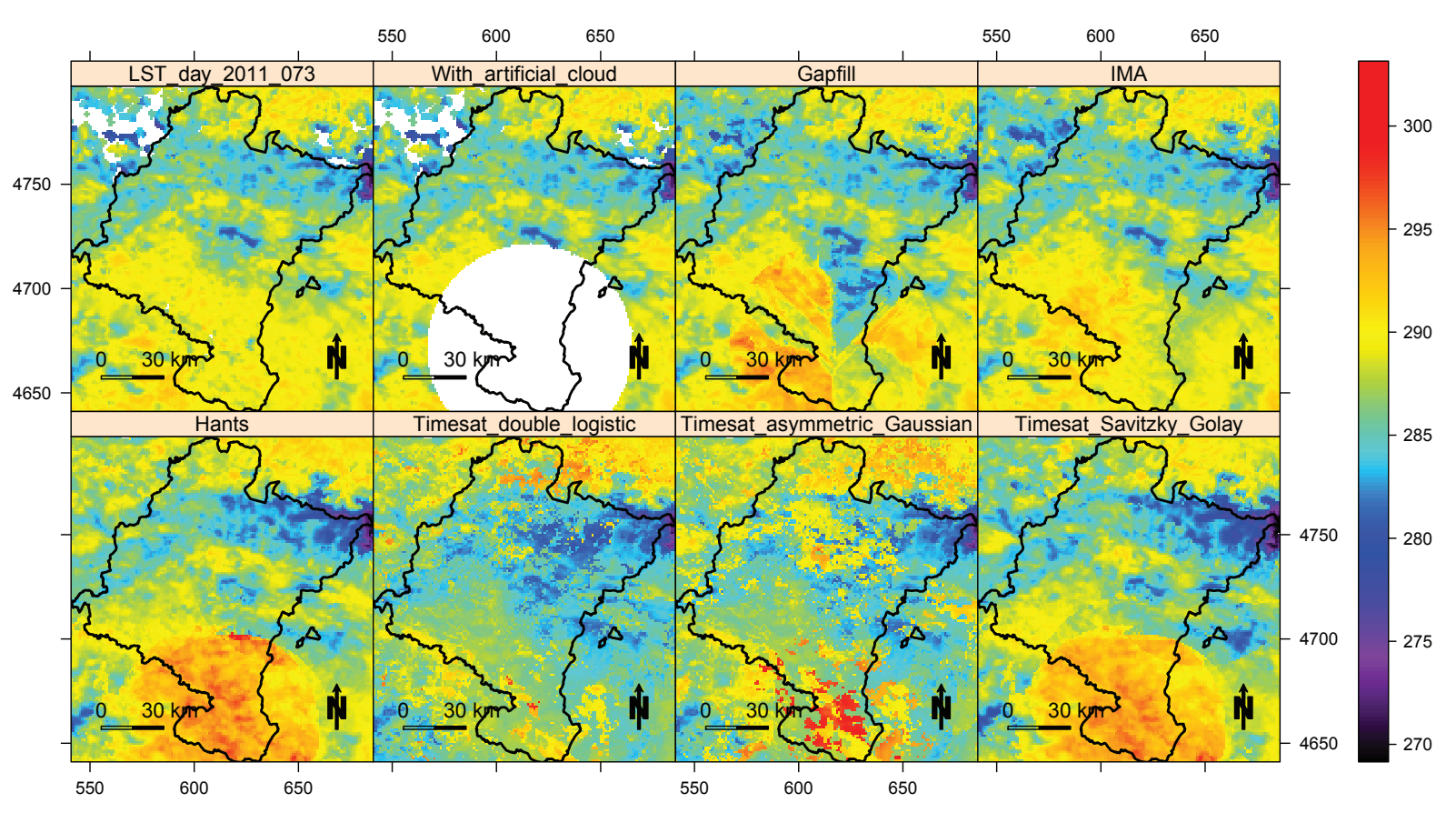

Fig. 7. LST_2011_073 daytime target image, the target image with artificial cloud, and the reconstructed images with Gapfill, IMA, Hants, Timesat double logistic, Timesat asymmetric Gaussian and Timesat Savitzky-Golay in Kelvin degrees.

TABLE VII

RUNNING TIMES IN MINUTES (M) AND HOURS (H) WHEN PROCESSING 138 LST DAY TIME SERIES OF $1 \mathrm{~km}^{2}$ RESOLUTION IMAGES IN NAVARRE WITH HANTS, THE TREE VERSIONS OF TIMESAT, GAPFILL AND IMA.

\begin{tabular}{|c|c|c|c|c|c|c|c|}
\hline & & \multirow{2}{*}{ Hants } & \multicolumn{3}{|c|}{ Timesat } & \multirow{2}{*}{ Gapfill } & \multirow{2}{*}{ IMA } \\
\hline & & & $\mathrm{DL}$ & $\mathrm{AG}$ & SG & & \\
\hline \multicolumn{2}{|c|}{ Pre-process } & $1 \mathrm{~m}$ & $2 \mathrm{~m}$ & $2 \mathrm{~m}$ & $2 \mathrm{~m}$ & $1 \mathrm{~m}$ & $0 \mathrm{~m}$ \\
\hline \multicolumn{2}{|c|}{ Configuration } & $5 \mathrm{~m}$ & $10 \mathrm{~m}$ & $10 \mathrm{~m}$ & $10 \mathrm{~m}$ & $0 \mathrm{~m}$ & $0 \mathrm{~m}$ \\
\hline \multirow{7}{*}{ 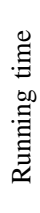 } & Type A & $2 \mathrm{~m}$ & $2 \mathrm{~m}$ & $2 \mathrm{~m}$ & $2 \mathrm{~m}$ & $27 \mathrm{~m}$ & $35 \mathrm{~m}$ \\
\hline & Type B & $2 \mathrm{~m}$ & $2 \mathrm{~m}$ & $2 \mathrm{~m}$ & $2 \mathrm{~m}$ & $59 \mathrm{~m}$ & $35 \mathrm{~m}$ \\
\hline & Type C & $2 \mathrm{~m}$ & $2 \mathrm{~m}$ & $2 \mathrm{~m}$ & $2 \mathrm{~m}$ & $1 \mathrm{~h} 3 \mathrm{~m}$ & $34 \mathrm{~m}$ \\
\hline & Type D & $2 \mathrm{~m}$ & $2 \mathrm{~m}$ & $2 \mathrm{~m}$ & $2 \mathrm{~m}$ & $2 \mathrm{~h} 10 \mathrm{~m}$ & $31 \mathrm{~m}$ \\
\hline & Type E & $2 \mathrm{~m}$ & $2 \mathrm{~m}$ & $2 \mathrm{~m}$ & $2 \mathrm{~m}$ & $11 \mathrm{~h} 20 \mathrm{~m}$ & $29 \mathrm{~m}$ \\
\hline & Type F & $2 \mathrm{~m}$ & $2 \mathrm{~m}$ & $2 \mathrm{~m}$ & $2 \mathrm{~m}$ & $20 \mathrm{~h} 2 \mathrm{~m}$ & $26 \mathrm{~m}$ \\
\hline & Type G & $2 \mathrm{~m}$ & $2 \mathrm{~m}$ & $2 \mathrm{~m}$ & $2 \mathrm{~m}$ & $1 \mathrm{~d} 6 \mathrm{~h} 13 \mathrm{~m}$ & $21 \mathrm{~m}$ \\
\hline \multicolumn{2}{|c|}{ Export to TIFF } & $2 \mathrm{~m}$ & $6 \mathrm{~m}$ & $6 \mathrm{~m}$ & $6 \mathrm{~m}$ & $1 \mathrm{~m}$ & $0 \mathrm{~m}$ \\
\hline \multicolumn{2}{|c|}{ Total time } & $21 \mathrm{~m}$ & $31 \mathrm{~m}$ & $31 \mathrm{~m}$ & $31 \mathrm{~m}$ & $2 \mathrm{~d} 17 \mathrm{~h} 16 \mathrm{~m}$ & $3 \mathrm{~h} 31 \mathrm{~m}$ \\
\hline
\end{tabular}

contiguous time periods across several years provides a more robust estimation of the mean. Anomalies need to be filtered because images are not always free of altered or distorted data. Shrinking spatial resolution of the filtered anomalies is also a necessary step to reduce the dimension of the equations to be solved in the thin-plate splines, and to mitigate the border effect. After interpolating the averaged anomalies, the new predictions are added to the mean image.

The IMA method is competitive for several reasons: a) it shows a strong agreement between the benchmark image and the filled image; $b$ ) it preserves the inherent phenology of the remote sensing data by estimating the mean of the same time periods in different years; c) neither the tuning constant nor any other parameter in the input configuration need to be specified in advance; d) its image-processing runtime is consistent regardless of the data-gap size to be filled; e) it exploits the benefits of the spatio-temporal dependence among time series of images, and f) it is easy to use. The simulation study reveals that IMA outperforms Timesat, Hants, and Gapfill, by reducing the RMSE for all three variables that were tested (LST Day, LST Night, and NDVI). The real case study also exhibits a good performance of IMA versus Gapfill, particularly when filling daily LST day images.

\section{ACKNOWLEDGEMENTS}

The authors would like to thank to the editor in chief Simon Yueh, and the comments made by the reviewers, leading to an improvement of our paper upon the original submission.

This research was supported by the project MTM2017-82553R (AEI/FEDER, UE), by the projects PI015-2016 and PI0432017 (Government of Navarre) and the Fundación CAN-Obra Social Caixa-UNED Pamplona 2016 and 2017.

\section{REFERENCES}

[1] Y. Gao, H. Xie, T. Yao, and C. Xue, "Integrated assessment on multitemporal and multi-sensor combinations for reducing cloud obscuration of MODIS snow cover products of the Pacific Northwest USA," Remote Sensing of Environment, vol. 114, no. 8, pp. 1662-1675, 2010.

[2] H. Xie, X. Wang, and T. Liang, "Development and assessment of combined Terra and Aqua snow cover products in Colorado Plateau, USA and northern Xinjiang, China," Journal of Applied Remote Sensing, vol. 3, no. 1, pp. 033 559-033 559, 2009. 
[3] G. Yang, H. Shen, L. Zhang, Z. He, and X. Li, "A moving weighted harmonic analysis method for reconstructing high-quality SPOT VEGETATION NDVI time-series data," IEEE Transactions on Geoscience and Remote Sensing, vol. 53, no. 11, pp. 6008-6021, 2015

[4] H. Müller, P. Rufin, P. Griffiths, A. J. B. Siqueira, and P. Hostert, "Mining dense Landsat time series for separating cropland and pasture in a heterogeneous Brazilian savanna landscape," Remote Sensing of Environment, vol. 156, pp. 490-499, 2015.

[5] A. Schneider, "Monitoring land cover change in urban and peri-urban areas using dense time stacks of Landsat satellite data and a data mining approach," Remote Sensing of Environment, vol. 124, pp. 689-704, 2012.

[6] J. G. Masek, E. F. Vermote, N. E. Saleous, R. Wolfe, F. G. Hall, K. F. Huemmrich, F. Gao, J. Kutler, and T.-K. Lim, "A Landsat surface reflectance dataset for North America, 1990-2000," IEEE Geoscience and Remote Sensing Letters, vol. 3, no. 1, pp. 68-72, 2006.

[7] J. G. Masek, S. N. Goward, R. E. Kennedy, W. B. Cohen, G. G. Moisen, K. Schleeweis, and C. Huang, "United States forest disturbance trends observed using Landsat time series," Ecosystems, vol. 16, no. 6, pp. 1087-1104, 2013.

[8] Q. Meng, B. E. Borders, C. J. Cieszewski, and M. Madden, "Closest spectral fit for removing clouds and cloud shadows," Photogrammetric Engineering \& Remote Sensing, vol. 75, no. 5, pp. 569-576, 2009.

[9] X. Zhu, F. Gao, D. Liu, and J. Chen, "A modified neighborhood similar pixel interpolator approach for removing thick clouds in Landsat images," IEEE Geoscience and Remote Sensing Letters, vol. 9, no. 3, pp. 521-525, 2012.

[10] T. Hermosilla, M. A. Wulder, J. C. White, N. C. Coops, and G. W. Hobart, "An integrated Landsat time series protocol for change detection and generation of annual gap-free surface reflectance composites," Remote Sensing of Environment, vol. 158, pp. 220-234, 2015.

[11] D. P. Roy, J. Ju, P. Lewis, C. Schaaf, F. Gao, M. Hansen, and E. Lindquist, "Multi-temporal MODIS-Landsat data fusion for relative radiometric normalization, gap filling, and prediction of Landsat data," Remote Sensing of Environment, vol. 112, no. 6, pp. 3112-3130, 2008.

[12] B. Chen, B. Huang, L. Chen, and B. Xu, "Spatially and temporally weighted regression: a novel method to produce continuous cloudfree Landsat imagery," IEEE Transactions on Geoscience and Remote Sensing, vol. 55, no. 1, pp. 27-37, 2017.

[13] L. Eklundh and P. Jönsson, "TIMESAT 3.2 with parallel processingSoftware Manual," Lund University, 2012

[14] W. Verhoef, M. Menenti, and S. Azzali, "Cover A colour composite of NOAA-AVHRR-NDVI based on time series analysis (1981-1992)," International Journal of Remote Sensing, vol. 17, no. 2, pp. 231-235, 1996.

[15] G. Roerink, M. Menenti, and W. Verhoef, "Reconstructing cloudfree NDVI composites using Fourier analysis of time series," International Journal of Remote Sensing, vol. 21, no. 9, pp. 1911-1917, 2000.

[16] F. Gerber, R. de Jong, M. E. Schaepman, G. Schaepman-Strub, and R. Furrer, "Predicting Missing Values in Spatio-Temporal Remote Sensing Data," IEEE Transactions on Geoscience and Remote Sensing, vol. 56, no. 5, pp. 2841-2853, 2018.

[17] F. Gerber, R. Furrer, G. Schaepman-Strub, R. de Jong, and M. E. Schaepman, "Predicting missing values in spatiotemporal satellite data," ArXiv e-prints, 2016. [Online]. Available: http://arxiv.org/abs/1605.01038

[18] M. Hutchinson and P. Gessler, "Splines more than just a smooth interpolator," Geoderma, vol. 62, no. 1-3, pp. 45-67, 1994.

[19] A. F. Militino, M. D. Ugarte, and U. Pérez-Goya, "Improving the Quality of Satellite Imagery Based on Ground-Truth Data from Rain Gauge Stations," Remote Sensing, vol. 10, no. 3, p. 398, 2018.

[20] J. C.-W. Chan, J. Ma, P. Kempeneers, and F. Canters, "Superresolution enhancement of hyperspectral CHRIS/Proba images with a thin-plate spline nonrigid transform model," IEEE Transactions on Geoscience and Remote Sensing, vol. 48, no. 6, pp. 2569-2579, 2010.

[21] X. Zhang, S. Liang, Z. Song, H. Niu, G. Wang, W. Tang, Z. Chen, and B. Jiang, "Local adaptive calibration of the satellite-derived surface incident shortwave radiation product using smoothing spline," IEEE Transactions on Geoscience and Remote Sensing, vol. 54, no. 2, pp. 1156-1169, 2016.

[22] Y. Jin, Y. Ge, J. Wang, Y. Chen, G. B. Heuvelink, and P. M. Atkinson, "Downscaling amsr-2 soil moisture data with geographically weighted area-to-area regression kriging," IEEE Transactions on Geoscience and Remote Sensing, vol. 56, no. 4, pp. 2362-2376, 2018.

[23] A. Verdin, C. Funk, B. Rajagopalan, and W. Kleiber, "Kriging and local polynomial methods for blending satellite-derived and gauge precipitation estimates to support hydrologic early warning systems,"
IEEE Transactions on Geoscience and Remote Sensing, vol. 54, no. 5, pp. 2552-2562, 2016.

[24] G. Matheron, "Splines and kriging: their formal equivalence," Down-toearth-statistics: Solutions looking for geological problems, pp. 77-95, 1981.

[25] M. L. Stein, "A kernel approximation to the kriging predictor of a spatial process," Annals of the Institute of Statistical Mathematics, vol. 43, no. 1, pp. 61-75, 1991.

[26] N. Cressie and C. K. Wikle, Statistics for spatio-temporal data. John Wiley \& Sons, 2015.

[27] R. E. Rossi, J. L. Dungan, and L. R. Beck, "Kriging in the shadows: geostatistical interpolation for remote sensing," Remote Sensing of Environment, vol. 49, no. 1, pp. 32-40, 1994.

[28] E. Addink, "A comparison of conventional and geostatistical methods to replace clouded pixels in NOAA-AVHRR images," International Journal of Remote Sensing, vol. 20, no. 5, pp. 961-977, 1999.

[29] J. N. Hird and G. J. McDermid, "Noise reduction of NDVI time series: An empirical comparison of selected techniques," Remote Sensing of Environment, vol. 113, no. 1, pp. 248-258, 2009.

[30] P. F. Velleman, "Definition and comparison of robust nonlinear data smoothing algorithms," Journal of the American Statistical Association, vol. 75, no. 371, pp. 609-615, 1980.

[31] M. Ma and F. Veroustraete, "Reconstructing pathfinder AVHRR land NDVI time-series data for the Northwest of China," Advances in Space Research, vol. 37, no. 4, pp. 835-840, 2006.

[32] D. Filipova-Racheva and M. Hall-Beyer, "Smoothing of NDVI time series curves for monitoring of vegetation changes in time," in Ecological monitoring and assessment network national science meeting, 2000, pp. 17-22.

[33] J. Zhou, L. Jia, and M. Menenti, "Reconstruction of global MODIS NDVI time series: Performance of Harmonic ANalysis of Time Series (HANTS)," Remote Sensing of Environment, vol. 163, pp. 217-228, 2015.

[34] L. Poggio, A. Gimona, and I. Brown, "Spatio-temporal MODIS EVI gap filling under cloud cover: An example in Scotland," ISPRS Journal of Photogrammetry and Remote Sensing, vol. 72, pp. 56-72, 2012.

[35] J. Chen, X. Zhu, J. E. Vogelmann, F. Gao, and S. Jin, "A simple and effective method for filling gaps in Landsat ETM+ SLC-off images," Remote Sensing of Environment, vol. 115, no. 4, pp. 1053-1064, 2011.

[36] M. Pringle, M. Schmidt, and J. Muir, "Geostatistical interpolation of SLC-off Landsat ETM plus images," ISPRS Journal of Photogrammetry and Remote Sensing, vol. 64, no. 1, pp. 654-664, 2009.

[37] X. Zhu, D. Liu, and J. Chen, "A new geostatistical approach for filling gaps in Landsat ETM+ SLC-off images," Remote Sensing of Environment, vol. 124, no. 1, pp. 49-60, 2012.

[38] G. Yin, G. Mariethoz, and M. F. McCabe, "Gap-Filling of Landsat 7 imagery using the direct sampling method," Remote Sensing, vol. 9, no. 1 , p. $12,2016$.

[39] C. Strong and K. M. Golden, "Filling the polar data gap in sea ice concentration fields using partial differential equations," Remote Sensing, vol. 8, no. 6, p. 442, 2016.

[40] P. M. Atkinson, C. Jeganathan, J. Dash, and C. Atzberger, "Intercomparison of four models for smoothing satellite sensor time-series data to estimate vegetation phenology," Remote Sensing of Environment, vol. 123, pp. 400-417, 2012.

[41] G. Yin, G. Mariethoz, Y. Sun, and M. F. McCabe, "A comparison of gap-filling approaches for Landsat-7 satellite data," International Journal of Remote Sensing, vol. 38, no. 23, pp. 6653-6679, 2017. [Online]. Available: https://doi.org/10.1080/01431161.2017.1363432

[42] R. Liu, R. Shang, Y. Liu, and X. Lu, "Global evaluation of gapfilling approaches for seasonal NDVI with considering vegetation growth trajectory, protection of key point, noise resistance and curve stability," Remote Sensing of Environment, vol. 189, pp. 164-179, 2017.

[43] J. M. Chen, F. Deng, and M. Chen, "Locally adjusted cubic-spline capping for reconstructing seasonal trajectories of a satellite-derived surface parameter," IEEE Transactions on Geoscience and Remote Sensing, vol. 44, no. 8, pp. 2230-2238, 2006.

[44] MODIS, "https://modis.gsfc.nasa.gov/about/," 2017. [Online]. Available: https://modis.gsfc.nasa.gov/about/

[45] D. A. Slayback, J. E. Pinzon, S. O. Los, and C. J. Tucker, "Northern hemisphere photosynthetic trends 1982-99," Global Change Biology, vol. 9, no. 1, pp. 1-15, 2003.

[46] C. J. Tucker, J. E. Pinzon, M. E. Brown, D. A. Slayback, E. W. Pak, R. Mahoney, E. F. Vermote, and N. El Saleous, "An extended AVHRR 8$\mathrm{km}$ NDVI dataset compatible with MODIS and SPOT vegetation NDVI data," International Journal of Remote Sensing, vol. 26, no. 20, pp. 4485-4498, 2005. 
[47] J. Rouse Jr, R. Haas, J. Schell, and D. Deering, "Monitoring vegetation systems in the Great Plains with ERTS," NASA special publication, vol. 351, p. 309, 1974

[48] M. T. van Wijk and M. Williams, "Optical instruments for measuring leaf area index in low vegetation: application in arctic ecosystems," Ecological Applications, vol. 15, no. 4, pp. 1462-1470, 2005.

[49] A. Benali, A. Carvalho, J. Nunes, N. Carvalhais, and A. Santos, "Estimating air surface temperature in Portugal using MODIS LST data," Remote Sensing of Environment, vol. 124, pp. 108-121, 2012.

[50] Z. Wan and J. Dozier, "A generalized split-window algorithm for retrieving land-surface temperature from space," IEEE Transactions on geoscience and remote sensing, vol. 34, no. 4, pp. 892-905, 1996.

[51] Z. Wan, Y. Zhang, Q. Zhang, and Z.-1. Li, "Validation of the land-surface temperature products retrieved from Terra Moderate Resolution Imaging Spectroradiometer data," Remote sensing of Environment, vol. 83, no. 1, pp. 163-180, 2002.

[52] S. N. Wood, "Thin plate regression splines," Journal of the Royal Statistical Society: Series B (Statistical Methodology), vol. 65, no. 1, pp. 95-114, 2003 .

[53] J. Li and A. D. Heap, "A review of comparative studies of spatial interpolation methods in environmental sciences: Performance and impact factors," Ecological Informatics, vol. 6, no. 3, pp. 228-241, 2011.

[54] W. Luo, M. Taylor, and S. Parker, "A comparison of spatial interpolation methods to estimate continuous wind speed surfaces using irregularly distributed data from England and Wales," International journal of climatology, vol. 28, no. 7, pp. 947-959, 2008.

[55] J. Duchon, "Splines minimizing rotation-invariant semi-norms in Sobolev spaces," Constructive theory of functions of several variables, pp. 85-100, 1977.

[56] E. P. Boer, K. M. de Beurs, and A. D. Hartkamp, "Kriging and thin plate splines for mapping climate variables," International Journal of Applied Earth Observation and Geoinformation, vol. 3, no. 2, pp. 146154, 2001.

[57] D. Nychka, R. Furrer, J. Paige, and S. Sain, "fields: Tools for spatial data," University Corporation for Atmospheric Research, Boulder, CO, USA, 2015, r package version 9.0. [Online]. Available: www.image.ucar.edu/fields

[58] M. Neteler and H. Mitasova, Open source GIS: a GRASS GIS approach. Springer Science \& Business Media, 2013, vol. 689.

[59] R Core Team, R: A Language and Environment for Statistical Computing, R Foundation for Statistical Computing, Vienna, Austria, 2018. [Online]. Available: https://www.R-project.org/

[60] C. Davino, M. Furno, and D. Vistocco, Quantile regression: theory and applications. John Wiley \& Sons, 2013.

[61] J. Leskovec, A. Rajaraman, and J. D. Ullman, Mining of massive datasets. Cambridge university press, 2014.

[62] A. Hadoop, "Hadoop," 2009.

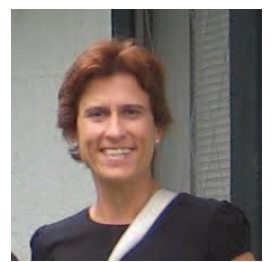

M Dolores Ugarte received the M.Sc. in Mathematics from the University of Zaragoza (Spain) in 1989, and the Ph.D. degree in Statistics at the Public University of Navarre (Spain) in 1996. Now she is full professor of Statistics at the Statistics, Computer Science and Mathematics Department at the Public University of Navarre, Spain. The research areas of interest are Spatial Statistics, Spatio-temporal Statistics, Remote Sensing, and Disease Mapping. She is the current co-editor in chief of the journal TEST, and associate editor of Statistical Modelling, and a member of the board of the Spatial and Spatio-Temporal Epidemiology. She is currently a member of the Executive Committee of the Federation of European National Statistical Societies (FenSTATs). She is coordinator of Mathematical projects in the Spanish Research Agency, and the principal investigator of several projects of the Spanish Ministry of Economy, Industry and Competitiveness.

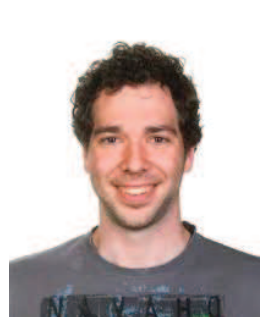

Unai Pérez-Goya received the BSc Computer Science Engineering at the University of Mondragón (Spain) in 2012. He received a Master in Innovation and Research in Informatics (MIRI) at the Polytechnic University of Catalonia, Barcelona (Spain) in 2015, and now he is Ph.D. student in Science and Industrial Technologies at the Public University of Navarra, Spain. His supervisors are Ana F. Militino and M. D. Ugarte.

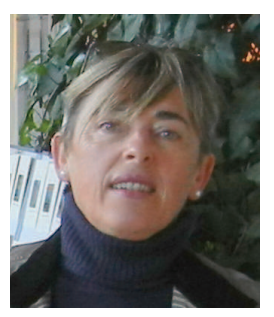

Ana F Militino received the M.Sc. in Mathematics from the University of Zaragoza (Spain) in 1981, and the Ph.D. degree in Statistics at the University of Extremadura (Spain), 1984. Now she is full professor of Statistics at the Statistics, Computer Science and Mathematics Department of the Public University of Navarre, Spain. The research areas of interest are Spatial Statistics, Spatio-temporal Statistics, and Remote Sensing where she has published numerous papers. In 2010 she received the John Griffiths teaching award by the International Association of Mathematical Geology. She coauthored the textbook Probability and Statistics with R published in CRC Press/Taylor and Francis in 2008 and a second edition in 2015. She has also participated in a large number of research projects sponsored by the Spanish Ministry of Education and Science, Agriculture, Navarre Statistical Institute, Basque Country Statistical Institute and other private companies.

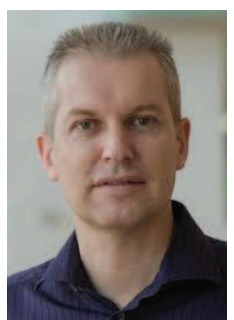

Marc Genton received the M.Sc. in Applied Mathematics from the Swiss Federal Institute of Technology (Switzerland) in 1992, and the Ph.D. degree in Statistics at the Swiss Federal Institute of Technology (EPFL), Lausanne. (Switzerland) in 1996. Now he is Distinguished Professor of Statistics, at the King Abdullah University of Science and Technology (KAUST, Saudi Arabia). He works on the statistical analysis, modeling, prediction, and uncertainty quantification of spatio-temporal data, with applications in environmental and climate science, renewable energies, geophysics, and marine science. 